\title{
The yeasts - versatile antimicrobial agents
}

\section{Ortansa Csutak ${ }^{\bowtie}$, Viorica Corbu}

Department of Genetics, Faculty of Biology, University of Bucharest, Bucharest, Romania

${ }^{凶}$ Correspondence: cs_ortansa@yahoo.fr

Received: 17 January 2020 / Revised: 17 April 2020 / Accepted: 23 April 2020 / Available online: 7 April 2020

Abstract Antagonistic yeasts present antimicrobial activity against a wide range of microorganisms, from other yeast species or strains, to bacteria and fungi from natural habitats, industrial processes or immunocompromised patients. The diversity of antimicrobial mechanisms depends mainly on the yeast species and might be represented by the production of killer toxins, enzymes and various cellular compounds or the presence of substrate competition mechanisms. The growing interest developed during last decades for the identification and characterization of yeasts with antimicrobial abilities, is related to their applications in biotechnology. The studies focus not only on understanding the genetic background of the antagonistic potential, but also on its improvement for controlling microbial contamination in natural and industrial fermentations, fruit decay and proliferation of pathogenic strains or biofilm formation on biomedical devices.

Keywords: yeasts, antimicrobial, biocontrol, biomedical

\section{Introduction}

Yeasts are ubiquitous microorganisms present in nature (soil, air, water, plants, animals, human, extreme environments) as well as in products related to human activities (fermented foods, feeds, waste waters, polluted sites) (Garcis-Bejar et al., 2019). Numerous yeast species comprise strains with specific genetic background correlated with various characteristics, from antimicrobial activity to production of biocompounds of large interest for biotechnology, medicine or environment protection.

During last decades there is a growing interest in using yeasts as an ecological solution to chemical agents extensively used in biomedicine, biocontrol or bioremediation. This is based on some important characteristics of the yeasts: (i) yeast strains with antagonistic abilities can be isolated from most ecosystems (Liu et al., 2013); (ii) the molecules involved in antimicrobial activities (killer toxins, pulcherrimin, carotenoid pigments) are not allergenic (Charoco et al., 2015) most of them having practical applications in more than one domain (e. g. biocontrol and biomedicine; biocontrol and bioremediation) (Younis et al., 2017); and last, but not least, (iii) many yeast strains have the ability to grow on cheap substrates, including those with polluting potential, producing high biomass yields (Abeln and Chuck, 2019). Due to their intensive use in production of fermented foods and beverages, some yeast species are considered as GRAS (Generally Regarded as Safe).
Many yeasts described so far do not have pathogenic potential and have a high metabolic versatility which recommends them for a large range of applications in industry.

The present review deals with the main aspects and the newest information concerning the mechanisms of the antimicrobial activity of the yeasts, its practical applications, as well as limitations and perspectives in various domains of modern biotechnology.

\section{Killer yeasts}

The killer phenotype of the yeasts was observed in the early 1960s at Saccharomyces cerevisiae strains able to excrete toxic proteins that produced the death of other microorganisms from the environment. Killer yeasts belong mainly to Saccharomyces, Hanseniaspora, Pichia, Zygosaccharomyces, Ustilago and Kluyveromyces genera, and can be isolated from fruits and plants, fermented foods (beer, wine, diary), soil or water. Also, some Candida and Issatchenkia strains were found to have killer activity, although there is few data concerning the toxins produced. The killer yeast strains are important not only in food production and preservation but also in biomedicine. Therefore, the studies regarding the characteristics, mechanism of action and genetic regulation of the killer toxins are of great interest for the scientific community. The $S$. cerevisiae strains can produce four types of killer toxins: K1, K2, K28 and Klus, encoded by ds-RNA genomes from viruslike particles found in the cytoplasm. 
The four killer phenotypes are classified based on their killing profile, cross-immunity and type of cell wall receptors recognized in the attacked cell. However, the K28 toxin is characteristic for the $S$. cerevisiae wine strain 28, and the Klus toxin was identified in the $S$. cerevisiae strains isolated from spontaneous fermentations of grapes from vineyards of the Ribera del Guadiana (Spain), showing amino acid sequence homolgy with a protein encoded by the chromosomal ORF YFRO20W (Rodriguez-Cousino et al., 2011; Becker and Schmitt, 2017). Lately, two killer toxins possibly encoded by chromosomal genes were isolated from $\mathrm{Cf} 8$ and M12 S. cerevisiae strains from wineries in Argentina, and a Kx killer factor able to inhibit growth of all known $S$. cerevisiae killer types was isolated from spontaneous fermentations of fruits and berries from Lithuania (Melvydas et al., 2016).

Species from Hanseniaspora, Zygosaccharomyces and Ustilago genera also present virus-like particles responsable for producing killer toxins with similar structure and mechansim of action as $S$. cerevisiae (Csutak, 2014).

The Pichia genus comprises species presenting a killer phenotype of great interest, in this case, the killer toxins being encoded by nuclear genes. Pichia anomala (Wickerhamomyces anomalus, Hansenula anomala) killer toxin is a glicoprotein with high exo- $\beta-1-3$ glucanase activity, Pichia kluyvery produces a toxin with a similar mode of action as $S$. cerevisiae K1 toxin (forming ion-permeable channels in the cell membrane of the sensitive cell), while Pichia membranefaciens toxin (PMKT2) seems to be induced by the presence of sodium chloride in the environment (Marquina et al., 2002).

Kluyveromyces species have two cytoplasmic linear DNA plasmids encoding the killer phenotype, the Kluyveromyces lactis killer toxin causing cell cycle arrest in the G1 phase in the sensitive cells.

Due to the broad range of microorganisms attacked (yeasts from the same species or other species/genera, bacteria, fungi) and to their different mechanism of action, the killer toxins are regarded as an ecological option for biocontrol and therapeutic applications. Thus, $S$. cerevisiae toxins suppressed the growth in vitro of Botrytis cinerea, while P. membranefaciens was able to protect Vitis vinifera plants against the same fungal infection (Chen et al., 2018). The activity of $P$. membranefaciens (PMKT2) and Ustilago maydis (KP6) toxins against the growth of Brettanomyces bruxellensis and Dekkera bruxellensis in wine production was found to be in close correlation with their concentration (Mehlomakulu et al., 2015).

The $P$. anomala killer toxin (PiKt) showed high activity against numerous Dekkera/Brettanomyces strains involved in wine spoilage (Padilla et al., 2018). The exo$\beta-1-3$ glucanases activity is responsible for the $P$. anomala antifungal activity against $B$. cinerea and Penicillium expansum on apples, B. cinerea on grapes, Penicillium digitatum on oranges and Colletotrichum gloeosporioides on papayas. This toxin produces emptied hyphae (B. cinerea), disruption of hyphal surfaces (Botryodiplodia theobromae) or growth inhibition (Penicillium roqueforti) (Muccilli and Restuccia, 2015).

The Kluyveromyces phaffii toxin (KpKt) is able to disrupt the integrity of the fungal cell wall, while the toxin produced by Kluyveromyces wickerhamii (KwKt) was effective against $B$. bruxellensis and $D$. bruxellensis had the ability to bind to the $\beta-1-6$-glucan receptors from the cell wall (Mehlomakulu et al., 2015).

Killer toxins are also intensively studied for their potential use in therapeutic applications. Thus, the possibility of successfully using $P$. anomala killer toxins for antimicrobial therapy, was demonstrated during experiments with anti-idiotypic antibodies for treatment of model candidiasis in rats (Passoth et al., 2006). Same toxins showed $\beta$-glucanase activity against Candida strains from mouth, bladder and skin infections. Pathogenic Candida strains were also inhibited by Zygosaccharomyces bailii toxin (zygocin). Bacterial pathogenic strains of Escherichia coli, Enterococcus faecalis, Klebsiella sp., Staphylococcus aureus, Pseudomonas aeruginosa and Pseudomonas alcaligenes were inhibited by Pichia kundravzevii killer toxin. Asynthetic decaPeptide derived from $P$. anomala killer toxin, named KP, had high antimicrobial activity against Candida albicans, Cryptococcus neoformans and Malassezia pachydermatis, while Z. bailii zygocin was active against $C$. albicans, Candida glabrata, Candida krusei and Sporothrix schenckii (Muccilli and Restuccia, 2015). Also, from the vaginal fluid of women infected with killer toxin sensitive microorganisms, human natural PaKT-like Abs ( $P a \mathrm{KTAbs}-P$. anomala Killer ToxinAntibodies) were isolated with protective role against Candida, Cryptococcus and Aspergillus infections (Polonelli et al., 2011).

\section{Substrate competition}

Competition for the nutrients and/or for space is one of the more effective mechanisms of antimicrobial action developed by few yeast species. In some cases, the antagonistic yeast is better adapted for surviving in certain environmental conditions compared to the competitor, has a better ability to colonize the surfaces or releases compounds that might act as chelators of various substrates.

Yeasts belonging to $S$. cerevisiae are well known for being effective in depleting sugars in fermented products. When inoculated during the first days of wine fermentation, S. cerevisiae has high antimicrobial activity reducing the number of other yeast species (Hanseniaspora uvarum, Candida zemplinina and Toluraspora delbrueckii) present at the end of the process (Lleixa et al., 2016). Sugar competition was also involved in antifungal activity of Sporobolomyces roseus, Rhodosporidium toruloides and Cryptococcus humicola against Botrytis cinerea and, respectively, the activity of 
Pichia guilliermondii against Ceratocystis paradoxa. On the other hand, $P$. expansum growth was inhibited due to nitrogen source competition in presence of $P$. guilliermondii and Candida sake (Muccilli and Restuccia, 2015).

In the case of $P$. guilliermondii, carbon and nitrogen substrate competition seems to be enhanced by the ability of the yeast cells to attach to the hyphae of $P$. digitatum, B. cinerea and Colletrotichum capsici (Papon et al., 2013). Also, P. guilliermondii colonize rapidly the wounds from the surface of fruits stimulating synthesis of enzymes (peroxidase, polyphenoloxidase, superoxide dismutase, catalase, phenylalanine ammonia-lyase) which correlates with an increasing of the plant defense mechanism (Zhao et al., 2008).

One of the best studied yeast species with applications in biocontrol is Metschnikowia pulcherrima. The antimicrobial mechanism of action is based on the competition for the iron ions from the environment (Saravanakumar et al., 2008). M. pulcherrima cells release the pulcherriminic acid (resulted from cyclodileucine, an intermediate in the L-leucine biosynthetic pathway) which chelates the iron $\mathrm{Fe}^{3+}$ forming the pigment pulcherrimin $\left(\mathrm{C}_{12} \mathrm{H}_{22} \quad \mathrm{Fe}_{2} \mathrm{~N}_{2} \mathrm{O}_{4}{ }^{2+}\right)$. The ability of $M$. pulcherrima of depleting iron from the growth media is correlated with the availability of $\mathrm{Fe}^{3+}$ and the ability of the competitive microorganisms to chelate $\mathrm{Fe}^{3+}$ (Sipiczki, 2006). Whole genome sequencing revealed that the gene $S N F 2$ regulates pulcherriminic acid biosynthesis and, thus, the antifungal activity in $M$. pulcherrima (Gore-Lloyd et al., 2018).

$M$. pulcherrima strains were very effective against $B$. cinerea, $P$. expansum, Alternaria sp., Monilia sp., Aspergillus carbonarius and Aspergillus niger, the pulcherrimin pigment affecting the fungal mycelium, the sporulation and formation of germination tubes (Piano et al., 1997; Spadaro et al., 2002; Bleve et al., 2006). Moreover, M. pulcherrima strains isolated from fruits were able to inhibit the growth of Candida pathogenic strains from human infections, the activity being augmented by the presence of sodium bicarbonate and calcium chloride (Csutak et al., 2013). In fact, using mixtures of yeasts inoculum and various chemicals (sodium bicarbonate, calcium chloride, ethanol or fungicides) in small concentrations is a successful approach in biocontrol applications (Karabulut et al., 2003).

Pulcherrimin production was also reported for other yeast species. Thus, Krause et al. (2018) described the structure and functions of the four genes (PUL1-4) forming a PULcherrimin (PUL) gene cluster present in K. lactis, Kluyveromyces aestuarii, Metschnikowia fructicola and Zygotorulaspora mraki. According to their study, PUL1 and PUL2 genes are involved in pulcherrimin biosynthesis, PUL3 encodes a membrane bound protein required for iron uptake from the medium using pulcherrimin and bringing the pigment-iron complexes into the cells (siderophore-like transporter mechanism), while PUL4 is involved in pulcherrimin biosynthesis as well as in $P U L 3$ positive regulation.

\section{Red yeasts}

The "red yeasts" represented by species belonging to the Phaffia, Rhodotorula, Sporobolomyces and Sporidiobolus genera, are able to synthesize carotenoid pigments, forming pink, orange or red colonies. The carotenoids are classified in hydrocarbon carotenes ( $\beta$-carotene, torulene) and oxygenated xanthophylls (torularhodin and astaxanthin). In present, there are known over 600 carotenoids produced by plants and microorganisms. The demand for these "natural" carotenoids is growing, being used as precursors of vitamin $\mathrm{A}$, in removing the oxygen radicals, as food colorants and antimicrobial agents.

Rhodotorula $\mathrm{sp}$. is able to produce relative high amounts of $\beta$-carotene, torulene and torularhodin using simple growth media, even industrial wastes (hydrocarbons, vegetal oils, glycerol). Carotenoid biosynthesis in Rhodotorula yeasts has been described. Whole genome sequencing studies of the strain Rhodotorula mucilaginosa RIT389 revealed the existence of a cluster consisting in three closely located genes encoding phytoene synthase $(\operatorname{crtB})$, lycopene cyclase $(\operatorname{crt} Y)$, and phytoene desaturase $(c r t I)$. The gene for geranyl pyrophosphate synthase has a separate location, while the genes $\operatorname{crt} X$ for carotenoid oxygenase and $\operatorname{crt} B Y$ for phytoene synthase/lycopene cyclase, are located in close proximity and are convergently transcribed (Tang et al., 2019). Landolfo et al. (2018) showed that in the strain $R$. mucilaginosa $\mathrm{C} 2.5 \mathrm{t} 1$, the genes for phytoene desaturase $(C A R 1)$, phytoene synthase/lycopene cyclase (CAR2) and carotenoid dioxygenase $(C A R O)$ are clustered in a genome region (CAR cluster) of approximately $10 \mathrm{~kb}$, and also, that the induction of genes involved in the early steps of carotenoid synthesis depends on the accumulation carotenoid pigment in the cells.

The carotenoid pigments extracted from Rhodotorula glutinis strains isolated from fruits, plants and soil had strong antibacterial effect against Staphylococcus aureus, Bacillus subtilis, Bacillus cereus, E. coli and Salmonella enteritidis (Keceli et al., 2013). Also, the pigment extracted from Sporobolomyces sp. had inhibitory activity against human pathogenic strains of $E$. coli and $S$. aureus (Manimala and Murugesan, 2014).

$R$. glutinis is also an effective biocontrol agent. The yeast cells were able to colonize rapidly the wounded fruits and inhibited the growth of $B$. cinerea and $P$. expansum on strawberries and apples by affecting spore germination (Zhang et al., 2007; Zhang et al., 2009).

The rhodotorulic acid produced by Rhodotorula species is a hydroxamate-type siderophore with high affinity for the $\mathrm{Fe}^{3+}$ ions from the environment. The antifungal effect of the rhodotorulic acid against $P$. expansum and $B$. cinerea constists in a delay of fungal installation in the host, respectively, inhibition of conidial growth, and is correlated with the iron concentration in the medium, the 
optimal activity being observed at $\mathrm{Fe}^{3+}$ concentrations up to $2 \mu \mathrm{M}$ (Ferramola et al., 2013).

Due to its importance and wide range of action, there are many studies regarding the possibility of enhancing the antimicrobial activity of Rhodotorula sp. For exemple, addition of $0.5 \%$ chitin in the growth media, augmented the effect of $R$. glutinis and $R$. mucilaginosa against $P$. expansum and Rhizopus sp. from peaches (Zhang et al., 2016). The antifungal activity of $R$. mucilaginosa against $P$. expansum was enhanced in combination with low concentrations of phytic acid which reduced the levels of patulin (Yang et al., 2015) and by heat shock treatments which determined a better yeast colonization on the surface of fruits (Cheng et al., 2016).

\section{Production of enzymes and molecules with antimicrobial activity}

\section{Volatile compounds}

Many yeast species produce a high number of volatile compounds (VOCs) with low molecular weight and high volatility, responsible mainly for the aroma of various final products. The VOCs are extensively used in the production of wine, fermented foods and feeds or in the flavor industry (for various cosmetics, chemicals or pharmaceuticals). Therefore, there are many studies dealing with the characterization of the yeast VOCs and their metabolic pathways. For example, S. cerevisiae strains involved in winemaking are able to produce 257 volatile metabolites represented by acetals, acids, alcohols, aldehydes, ketones, terpenic compounds, esters, ethers, furan-type compounds, hydrocarbons, pyrans, pyrazines and S-compounds related to different metabolic pathways. Thus, the aliphatic acids with short carbon chain result from fermentative metabolism, the mediumcarbon-chain fatty are intermediates in fatty acid biosynthesis while the higher alcohols are produced by the Ehrlich pathway and/or by the pyruvate pathway (Alves et al., 2015).

Some VOCs, such as esters, alcohols and sulfides, have antimicrobial activity against a wide range of phytopathogenic fungi. Since VOCs are considered as ecofriendly compounds, they might represent an interesting alternative in biocontrol for preventing plants and fruits decay. One of the yeast species producing VOCs with antifungal activity is Candida sake. The strains of $C$. sake are able to synthesize alcohols, from which 3,7-Dimethyl-6-octen-1-ol (citronellol) and phenylethyl alcohol with antifungal activity against dermatophytes and Aspergillus sp., and phenylethyl alcohol effective against Muscodor albus (Arrarte et al., 2017). C. sake also produces esters (ethyl hexanoate, 3methylbutyl hexanoate and 2-phenylethyl acetate) with antifungal activity.

Other Candida species producing VOCs are Candida intermedia and Candida friedrichii. C. intermedia along with Sporidiobolus pararoseus, $W$. anomalus (H. anomala, $P$. anomala) and $M$. pulcherrima VOCs inhibited spore germination and mycelial growth of $B$. cinerea (Arrarte et al., 2017). The compounds synthesized by $C$. friedrichii as well as $C$. intermedia, Cyberlindnera jadinii and Lachancea thermotolerans determined the reduction of vegetative growth, sporulation and production of ochratoxin A (OTA) by Aspergillus carbonarius and Aspergillus ochraceus (Arrarte et al., 2017; Farbo et al., 2018). In this case, the main compound of yeast VOCs was 2-phenylethanol, an alcohol with high antifungal activity. For example, the 2phenylethanol from $S$. cerevisiae was observed to control Sclerotinia sclerotiorum growth in vitro and in bean seeds and the one produced by Kloeckera apiculata was active against Penicillium italicum mold in citrus fruit. The $P$. anomala ( $W$. anomalus) 2-phenylethanol inhibited spore germination and aflatoxin B1 production by Aspergillus flavus in correlation with down regulation of aflatoxin biosynthesis genes and an altered expression of chromatin modifying genes (Hua et al., 2014). Also, the VOCs from $P$. anomala (W. anomalus), Pichia kluyveri and $H$. uvarum were described as active against $A$. ochraceus growth and OTA synthesis during processing of Coffea arabica (Farbo et al., 2018).

The increase of ethyl acetate concentration as a result of glucose metabolism in P. anomala (W. anomalus) cells, determined the decrease of Penicillium roqueforti growth (Passoth et al., 2006). Ethyl acetate, acetate, acetic acid and hydrogen sulfide produced by $H$. uvarum, $S$. cerevisae, and Meyerozyma (Candida, Pichia) guilliermondii were also responsible for inhibiting $B$. cinerea development on Vitis vinifera berries (CorderoBueso et al., 2017).

Less studied yeast species such as Aureobasidium pullulans or Galactomyces candidum also showed high rates of $P$. expansum and $B$. cinerea inhibition using VOCs (Chen et al., 2018).

\section{Cell wall-lytic enzymes and proteases}

Cell wall-lytic enzymes (chitinases, $\beta$-glucanases) and proteases with antimicrobial activity are produced by yeast species belonging mainly to Aerobasidium, Pichia, Hanseniaspora, Metschnikowia and Rhodotorula genera. The antimicrobial activity of the chitinases is based on their ability to hydrolyze the $\beta-(1-4)$ linkages in the chitin and is related to the fact that filamentous fungi present more than $20 \%$ chitin in their cell wall and hyphae (Hartl et al., 2012). The $\beta$-glucanases involved in antimicrobial activity are mostly exo- $\beta-1-3$-glucanases that break $\beta-1$ 3 -glucans, while the proteases are involved in various physiological and metabolic functions from cell division and signal transduction to apoptosis.

A. pullulans is one of the species able to produce chitinases, CMCase (carboxymethyl cellulase), pectinases and $\beta$-1,3-glucanases (responsible for hydrolyzing the laminarin in fungal cell wall) (Chen et al., 2018). Some strains produced an extracellular alkaline serine protease of 415 amino-acids $(\mathrm{M}=42.9 \mathrm{kDa})$ encoded by the gene ALP5, which inhibited the mycelia growth of Monilia 
laxa on plums, peaches and stone fruits, and B. cinerea and $P$. expansum on apples and pomme fruits (Zhang et al., 2012).

$M$. pulcherrima and $M$. fructicola strains were able to secrete high levels of chitinases in presence of cell wall fractions of B. cinerea, respectively, Monilinia fructicola. The $M$. fructicola chitinase is encoded by MfChi gene, has 365 amino and a molecular weight of $40.9 \mathrm{kDa}$, and a recombinant protein obtained in Pichia pastoris cells showed high impact on fungi spore germination and germ tube length (Banani et al., 2015).

$R$. mucilaginosa produced lytic enzymes active against Colletotrichum gloeosporioides from papaya, while Rhodotorula minuta and S. cerevisiae were able to produce $\beta$-1-3-glucanases. Sometimes, the ability of yeast species to act as antimicrobial agents is based on the synthesis of more than one type of lytic enzymes (chitinases, $\beta-1,3$ and $\beta-1,6$-glucanases, proteases or mannanases) which perform thus a more efficient attack against the cell wall of phytopathogenic fungi. For exemple, multiple lytic enzymes were described as responsible for the activity of $R$. mucilaginosa and Candida famata against $C$. gloeosporioides producing postharvest anthracnose in papaya fruits (Muccilli and Restuccia, 2015; Ferazz et al., 2016).

Pichia guilliermondii strains revealed chitinase and $\beta$ 1,3-glucanase activity against $B$. cinerea when grown in presence of fungal cell wall and sucrose or glucose (Ferazz et al., 2016). In vitro studies using a recombinant $P$. guilliermondii $\beta-1,3$-glucanase and $E$. coli host cells, showed that the main mechanism of action relies in reduction of $B$. cinerea germ tube length (Papon et al., 2013). Also, several strains of $P$. guilliermondii were described as biocontrol agents against $P$. expansum, $P$. italicum, $P$. digitatum, B. cinerea, C. capsici, Rhizopus stolonifer, Rhizopus nigricans and Botryodiplodia theobromae. $P$. guilliermondii and $P$. membranefaciens also had $\beta$-1,3-glucanase and chitinase activities against $R$. stolonifer on harvested nectarine and peach fruits (Fan et al., 2002).

\section{Biosurfactants}

The biosurfactants are amphiphilic compounds produced by different microbial strains in order to increase the solubility of hydrophobic compounds. Such compounds are widely used in bioremediation, agriculture, food and pharmaceutical industry mainly due to their safety characteristics. The biosurfactants have low toxicity, high biodegradability and high stability in stress conditions induced by variation of physical parameters such as $\mathrm{pH}$, temperature, ionic strength (Fakruddin 2012). These unique metabolic molecules produced by a wide range of microorganism present both hydrophilic and hydrophobic moieties being able to reduce surface/interfacial tension. Many yeasts species such as Yarrowia lipolytica, Candida apicola, C. tropicalis, Starmerella bombicola, Pseudozyma antarctica, are able to synthetize biosurfactants as an efficient mechanism for adapting to growth conditions. Although at the beginning biosurfactants were mainly used in bioremediation processes, recently they became an important area of interest for biomedical purpose due to their ability to act as anti-adhesive and antimicrobial agents.

Sophorolipids (SLP), a major class of biosurfactants, are produced as a mixture of structurally related molecules (lactonic and acidic form) synthetized by several yeast species (C. bombicola (de Rientso et al., 2015), Candida. batistae (Konishi et al., 2008), Candida riodocensis (Kurtzman et al., 2010), Candida kuoi (Kurtzman et al., 2012), P. anomala (Thaniyavarn et al., 2008), Cyberlindnera samutprakarnensis (Poomtien et al., 2013) and Wickerhamiella domercqiae (Chen et al., 2006)). The lactonic form of SLP exhibit biocidal activities (being intensively studied for their biomedical potential) while the acidic form is a better foaming agent (with potential use in food and cosmetic industries). The biocidal activity of SLP is due to their property to destabilize and to alter the permeability of the cellular membrane of microbial cells. Depending on the chemical structure of the wall of the pathogenic microorganisms and the composition of the obtained sophorolipids mixture, different degrees of inhibition were observed (de Oliveira et al., 2015; Archana et al., 2019). The acetylated lactonic form of SLP inhibits growth of Gram positive bacteria such as $B$. subtilis, $S$. epidermidis, Streptococcus faecium and Propionibacterium acnes. Lauryl alcohol-derived sophorolipids inhibit growth of pathogenic Gram negative bacteria (E. coli, $P$. aeruginosa) by causing the cells shrinking or by inducing irregularities on the cell surface, and of Gram positive bacteria ( $S$. aureus and $B$. subtilis) by destabilizing the bacterial wall (DenglePulate et al., 2014). Other studies have shown that this type of SLP can be used as adjuvants in antibiotics treatment by enhancing their activity. SLP also increase antifungal activity of polyhexamethylene biguanide, a polymer used as disinfectant for preventing Trichophyton rubrum, Trichophyton mentagrophytes and Tinea pedis infections (Sanada et al., 2014).

Mannosylerythritol lipids (MELs) represent a different class of biosurfactants produced by Ustilago and Pseudozyma strains. Depending on the number of acetyl groups attached to the hydrophilic moiety MELs were separated into 4 main categories. Of these, MELs A and $B$ exhibits high antibacterial activity against a variety of Gram positive bacteria but week activity against Gram negative bacteria. A study conducted by Nashida et al., (2018) showed that different MELs with 4-O- $\beta$-Dmannopyranosyl-D-erythritol structures obtained at lab scale exhibit high antimicrobial activity against multidrug-resistant bacteria such as methicillin-resistant $S$. aureus and vancomycin resistant Enterococci (E.faecalis and E. faecium).

A worldwide challenge is represented by the pathogenic microbial biofilm that is a consortium of microbial cells preserved by a polymeric matrix which acting as a barrier against chemical antimicrobial drugs and the protective 
mechanisms of the host immune systems (Paraszkiewicz et al., 2019). The formation of biofilms usually starts as soon as a biomedical device (catheters, prosthesis) is implanted in the body of a patient exposing him to the risk of getting a difficult to treat infection (Epstain et al., 2011; Satpute et al., 2016). Several studies have reported that biosurfactants can be very effective as biofilm formation inhibitors or as agents for controlling/disruption of already existent biofilms (Banat et al., 2014).

Although the mechanism of anti-adhesive action of biosurfactants is not well understood it seems that the adsorption of biosurfactants on the abiotic surface changes its hydrophobicity interfering thus with the early stages of biofilm formation. Biosurfactants produced by Candida sphaerica, lunasan (Luna et al., 2011), showed antimicrobial activity against Streptococcus oralis, Staphylococcus epidermidis and Candida albicans and antiadhesive activity against $P$. aeruginosa, Streptococcus agalactiae and Streptococcus sanguis. Also rufisan, a biosurfactant produced by $Y$. lipolytica, is an effective anti-adhesive biocompound against Streptococcus mutans, $S$. aureus and $S$. agalactiae (Rufino et al., 2013).

\section{Present limitations and future prospective}

The interest in using yeasts as antimicrobial agents for biocontrol and biomedical applications implies a deeper knowledge on the limitations and perspectives of the processes.

In present, a number of compounds comprising yeasts with antifungal activity are produced in order to control different phytopathogens, such as gray mold caused by $B$. cinerea: Shemer (based on $M$. fructicola), produced by Bayer/Koppert Biological Systems (Neteherlands, Germany), Candifruit (based on $C$. sake) produced by IRTA (Spain), Boni protect/Botector (based on $A$. pullulans) produced by Bio-Ferm/Manica (Austria) or Nexy (based on Candida oleophila) produced by Lasaffre (France) (Romanazzi et al., 2016).

Although using yeast as biocontrol agents might represent an ecological solution for the fungicides, there are still some problems to be solved. For instance, in order to be efficient, the biocontrol agent must be applied as soon as possible after fruits harvesting, on fruits with no pre-existing infections, preserved in a proper environment. Also, the concentration of the agents and their storage are important factors. The antagonistic agents act on the surface of the plants/fruits in a microenvironment, in correlation with other microorganisms representing the native microbiota, their activity being influenced by the climatic conditions.

In order to augment the biocontrol effect of the yeasts, the surface of the plants/fruits can undergo thermal, chemical or radiation treatments and various compounds (salts, alcohols, surfactants) can be added in order to modify the $\mathrm{pH}$ and nutritional substrate of the infection site or to enhance the antimicrobial activity of the yeasts. Thus, sodium bicarbonate was used to enhance the antifungal activity of Cryptococcus laurentii and Trichosopron pullulans (Yao et al., 2004), sodium bicarbonate and calcium chloride augmented the antimicrobial activity $M$. pulcherrima against fungi as well as human pathogenic Candida strains (Csutak et al., 2013), while microwave treatements of the wounds on the surface of the fruits also had a positive influence on the antifungal activity of $M$. pulcherrima against Penicillium citrinum (Guo et al., 2016). Ochratoxin synthesis by $A$. ochraceus was highly reduced when the fungus was exposed to volatile compunds produced by $P$. anomala, $P$. kluyveri and $H$. uvarum grown on malt yeast glucose peptone medium (Masoud et al., 2005). The accurate identification of the volatile compounds responsible for the antimicrobial activity and the optimization of synthesis parameters are necessary in order to enhance the impact of these compounds on phytopathogens and also for obtaining artificial mixtures of volatile compounds with high efficiency and nonspecific reduced (Farbo et al., 2018).

In what concerns the killer toxins, they are very sensitive to environmental conditions, most of them acting at acid $\mathrm{pH}$ values and approximately $20^{\circ} \mathrm{C}$. Therefore, their use in medicine implies special requirements. However, addition of killer toxins to buffered solutions is used for treatment of skin and mucosal membranes candidiasis (Marquina et al., 2002). Due to their antigenic properties, the killer toxins cannot be used as antibiotics, but antiidiotypic antibodies were obtained, presenting similar effect against $C$. albicans infections as $P$. anomala killer toxins. The growth media might also influence the production of killer toxins, the addition of glucose augmenting the production of killer toxins in $P$. anomala (Passoth et al., 2006).

On the other side, the production of carotenoids in "red yeasts" is influenced by the temperature and regulated by light and presence of metal ions, and can be improved by UV or chemical mutagenesis (Tang et al., 2019).

\section{Conclusions}

Antimicrobial activity against human or food borne pathogens is a remarkable feature of some yeasts recommending them for biocontrol or biomedical use. The diversity of mechanisms (killer toxin production, substrate competition, secondary metabolites production such as: carotenoids, pigments, biosurfactants, volatile compounds, enzymes) involved in the antimicrobial activity of yeasts represent an advantage for using these microorganisms in developing new bio-based products for food preservation, biocontrol or as new drugs for human infections treatment.

In present, the pollution level has reached highest rates. As a consequence, most of the legal provisions are directed towards finding ecological alternatives for the chemicals used in the numerous industrial processes. 
Although there are many problems waiting to be solved prior using yeasts as antimicrobial agents, there are numerous yeast based products already commercialized and successfully used for food preservation or crops biocontrol. Another problem of humanity is the inability to combat infections caused by multidrug resistant microorganisms. Since a mechanism of antibioticresistant gene transfer between yeasts and other microorganisms has not yet been described, yeasts might represent the safest alternative for probiotic development or growth inhibition of human pathogens. Therefore, the study of yeasts with antimicrobial potential is and will remain a priority research direction.

\section{(C) The Author(s) 2020}

Open Access This article is distributed under the terms of the Creative Commons Attribution 4.0 International License (http://creativecommons.org/licenses/by/4.0/) which permits unrestricted use, distribution, and reproduction in any medium, provided you give appropriate credit to the original author(s) and the source, provide a link to the Creative Commons license, and indicate if changes were made.

\section{References}

Abeln F., Chuck C.J. 2019. Achieving a high density oleaginous yeast culture: comparison of four processing strategies using Metschnikowia pulcherrima. Biotechnol. Bioeng. 116, 3200-3214.

Alves Z., Melo A., Figueiredo A.R., Coimbra M.A., Gomes A.C., Rocha S.M. 2015. Exploring the Saccharomyces cerevisiae volatile metabolome: indigenous versus commercial strains. PLoS One 10. doi: 10.1371/journal.pone.0143641.

Archana K., Reddy K.S., Parameshwar J., Bee H. 2019. Isolation and characterization of sophorolipid producing yeast from fruit waste for application as antibacterial agent. Environ. Sustain. 2, 107-115.

Arrarte E., Garmendia G., Rossini C., Wisniewski M., Vero S. 2017. Volatile organic compounds produced by Antarctic strains of Candida sake play a role in the control of postharvest pathogens of apples. Biol. Control 109, 14-20.

Banani H., Spadaro D., Zhang D., Matic S., Garibaldi A., Gullino M.L. 2015. Postharvest application of a novel chitinase cloned from Metschnikowia fructicola and overexpressed in Pichia pastoris to control brown rot of peaches. Int. J. Food Microbiol. 199, 54-61.

Banat I.M, Diaz De Rienzo M.A., Quinn G.A. 2014. Microbial biofilms: biosurfactants as antibiofilm agents. Appl. Microbiol. Biotechnol. 98, 9915-9929.

Becker B., Schmitt M. 2017. Yeast killer toxin K28: biology and unique strategy of host cell intoxication and killing. Toxins 9, 333-348.

Bleve G., Grieco F., Cozzi G., Logrieco A., Visconti A. 2006. Isolation of epiphytic yeasts with potential for biocontrol of Aspergillus carbonarius and A. niger on grape. Int. J. Food Microbiol. 108, 204-209.
Carocho M., Morales P., Ferreira I.C. 2015. Natural food additives: Quo vadis? Trends Food Sci. Tech. 45, 284295.

Chen J., Song X., Zhang H., Qu Y. 2006. Production, structure elucidation and anticancer properties of sophorolipid from Wickerhamiella domercqiae. Enzyme Microb. Technol. 39, 501-506.

Chen P.H., Chen R.Y., Chou J.Y. 2018. Screening and evaluation of yeast antagonists for biological control of Botrytis cinerea on strawberry fruits. Mycobiology. 46, 33-46.

Cheng Z., Chi M., Li G., Chen H., Sui Y., Sun H., Liu J. 2016. Heat shock improves stress tolerance and biocontrol performance of Rhodotorula mucilaginosa. Biol. Control. 95, 49-56.

Cordero-Bueso G., Mangieri N., Maghradze D., Foschino R., Valdetara F., Cantoral J.M., Vigentini I. 2017. Wild grape-associated yeasts as promising biocontrol agents against Vitis vinifera fungal pathogens. Front. Microbiol. 8, 2025-2035.

Csutak O. 2014. Genetica şi biodiversitatea drojdiilor cu aplicaţii biotehnologice, Ed. Universităţii din Bucureşti.

Csutak O., Vassu T., Sarbu I., Stoica I., Cornea P. 2013. Antagonistic activity of three newly isolated yeast strains from the surface of fruits, Food Technol. Biotech. 51, 70-77.

de Oliveira M.R., Magri A., Baldo C., Camilios-Neto D., Minucelli T., Celligoi M.A.P.C. 2015. Sophorolipids a promising biosurfactant and its applications. Int. J. Adv. Biotechnol. Res. 6, 161-174.

de Rienzo M.A.D., Banat I.M., Dolman B., Winterburn J., Martin P.J. 2015. Sophorolipid biosurfactants: possible uses as antibacterial and antibiofilm agent. New Biotechnol. 32, 720-726.

Dengle-Pulate V., Chandorkar P., Bhagwat S., Prabhune A.A. 2014. Antimicrobial and SEM studies of sophorolipids synthesized using lauryl alcohol. $J$. Surfactants Deterg. 17, 543-552.

Epstein A.K., Pokroy B., Seminara A., Aizenberg J. 2011. Bacterial biofilm shows persistent resistance to liquid wetting and gas penetration. Proc. Natl. Acad. Sci. 108, 995-1000.

Fakruddin MD., 2012. Biosurfactant: production and application. J. Pet. Environ. Biotechnol. 3, 2-7.

Fan Q., Tian S., Liu H., Xu Y. 2002. Production of $\beta$-1, 3-glucanase and chitinase of two biocontrol agents and their possible modes of action. Chin. Sci. Bull. 47, 292. doi:10.1360/02tb9070

Farbo M.G., Urgeghe P.P., Fiori S., Marcello A., Oggiano S., Balmas V., Hassan Z.U., Jaoua S., Migheli Q. 2018. Effect of yeast volatile organic compounds on ochratoxin A-producing Aspergillus carbonarius and A. ochraceus. Int. J. Food Microbiol. 284, 1-10.

Ferramola M.S., Benuzzi D., Calvente V., Calvo J., Sansone G., Cerutti S., Raba J. 2013. The use of siderophores for improving the control of postharvest 
diseases in stored fruits and vegetables. In Microbial Pathogens and Strategies for Combating Them, Mendez-Vila A. (Ed.), Science Technology and Education, Formatex Research Center Spain, 13851394.

Ferraz L.P., da Cunha T., da Silva A.C., Kupper K.C. 2016. Biocontrol ability and putative mode of action of yeasts against Geotrichum citri-aurantii in citrus fruit. Microbiol.Res. 188, 72-79.

García-Béjar B., Fernández-Pacheco P., Briones A., Arévalo-Villena M. 2019. Yeast from distillery plants: a new approach. In: Advances in Grape and Wine Biotechnology. IntechOpen. doi: 10.5772/intechopen.86291

Gore-Lloyd D., Sumann I., Brachmann A.O., Schneeberge, K., Ortiz-Merino R.A., Moreno-Beltran M., Schläfli M., Kirner P., Santos A., Freimoser M. 2018. Snf2 controls pulcherriminic acid biosynthesis and connects pigmentation and antifungal activity of the yeast Metschnikowia pulcherrima. bioRxiv 494922-494942.

Guo D., Yang B., Ren X., Zhu L. 2016. Effect of an antagonistic yeast in combination with microwave treatment on postharvest blue mould rot of jujube fruit. J. Phytopathol. 164, 11-17.

Hartl L., Zach S., Seidl-Seiboth V. 2012. Fungal chitinases: diversity, mechanistic properties and biotechnological potential. Appl. Microbiol. Biotechnol. 93, 533-543.

Hua S.S.T., Beck J.J., Sarreal S.B.L., Gee W. 2014. The major volatile compound 2-phenylethanol from the biocontrol yeast, Pichia anomala, inhibits growth and expression of aflatoxin biosynthetic genes of Aspergillus flavus. Mycotoxin Res. 30, 71-78.

Karabulut O.A., Smilanick J.L., Gabler F.M., Mansour M., Droby S. 2003. Near-harvest applications of Metschnikowia fructicola, ethanol, and sodium bicarbonate to control postharvest diseases of grape in central California, Plant Dis. 87, 1384-1389.

Keceli T.M., Erginkaya Z., Turkkan E., Kaya U. 2013. Antioxidant and antibacterial effects of carotenoids extracted from Rhodotorula glutinis strains. Asian J. Chem. 25, 1-5.

Konishi M., Fukuoka T., Morita T., Imura T., Kitamoto D. 2008. Production of new types of sophorolipids by Candida batistae. J. Oleo Sci. 57, 359- 369.

Kot A.M., Błażejak S., Gientka I., Kieliszek M., Bryś J. 2018. Torulene and torularhodin:"new" fungal carotenoids for industry?. Microb Cell Fact. 17, 4956.

Krause D.J., Kominek J., Opulente D.A., Shen X.X., Zhou X., Langdon Q.K., DeVirgiliof J., Hulfachora A.B., Kurtzmanf C.P., Hittinger C.T. 2018. Functional and evolutionary characterization of a secondary metabolite gene cluster in budding yeasts. Proc. Natl. Acad. Sci. 115, 11030-11035.

Kurtzman C.P. 2012. Candida kuoi sp. nov., an anamorphic species of the Starmerella yeast clade that synthesizes sophorolipids. Int. J. Syst. Evol. Microbiol. 62, 2307-2311

Kurtzman C.P., Price N.P.J., Ray K., Kuo T.M. 2010. Production of sophorolipid biosurfactants by multiple species of the Starmerella (Candida) bombicola yeast clade. FEMS Microbiol. Lett. 311, 140-146.

Landolfo S., Ianiri G., Camiolo S., Porceddu A., Mulas G., Chessa R., Giacomo Z., Mannazzu I. 2018. CAR gene cluster and transcript levels of carotenogenic genes in Rhodotorula mucilaginosa. Microbiol. 164, 78-87.

Liu J., Sui Y., Wisniewski M., Droby S., Liu Y. 2013. Utilization of antagonistic yeasts to manage postharvest fungal diseases of fruit. Int. J. Food Microbiol. 167, 153-160.

Lleixà,J., Manzano M., Mas A., Portillo M.D.C. 2016. Saccharomyces and non-Saccharomyces competition during microvinification under different sugar and nitrogen conditions. Front Microbiol. 7, 1959-1969.

Luna J.M., Rufino R.D., Sarubbo L.A., Rodrigues L.R., Teixeira J.A., de Campos-Takaki G.M. 2011. Evaluation antimicrobial and antiadhesive properties of the biosurfactant Lunasan produced by Candida sphaerica UCP 0995. Curr. Microbiol.62, 15271534.

Manimala M.R.A., Murugesan R. 2014. In vitro antioxidant and antimicrobial activity of carotenoid pigment extracted from Sporobolomyces sp. isolated from natural source. J. Appl. Nat. Sci. 6, 649-653.

Marquina D., Santos A., Peinado J. 2002. Biology of killer yeasts. Int. Microbiol. 5, 65-71.

Masoud W., Poll L., Jakobsen M. 2005. Influence of volatile compounds produced by yeasts predominant during processing of Coffea arabica in East Africa on growth and ochratoxin A (OTA) production by Aspergillus ochraceus. Yeast 22, 1133-1142.

Md F. 2012. Biosurfactant: production and application. $J$. Pet. Environ. Biotechnol. 3, 2-7.

Mehlomakulu N.N., Setati M.E., Divol B. 2015. NonSaccharomyces killer toxins: Possible biocontrol agents against Brettanomyces in wine? S. Afr. J. Enol. 36, 94-104.

Melvydas V., Bružauskaitė I., Gedminienė G., Šiekštelè, R. 2016. A novel Saccharomyces cerevisiae killer strain secreting the $\mathrm{X}$ factor related to killer activity and inhibition of S. cerevisiae K1, K2 and K28 killer toxins. Indian J. Microbiol. 56, 335-343.

Muccilli S., Restuccia C. 2015. Bioprotective role of yeasts. Microorganisms 3, 588-611.

Nashida J., Nishi N., Takahashi Y., Hayashi C., Igarashi M., Takahashi D., Toshima, K. 2018. Systematic and stereoselective total synthesis of mannosylerythritol lipids and evaluation of their antibacterial activity. $J$. Org. Chem. 83, 7281-7289

Padilla B., Gil J., Manzanares P. 2018. Challenges of the non-conventional yeast Wickerhamomyces anomalus in winemaking. Fermentation 4, 68-82. 
Papon N., Savini V., Lanoue A., Simkin A.J., Crèche J., Giglioli-Guivarc'h N., Courdavault V., Sibirny A. 2013. Candida guilliermondii: biotechnological applications, perspectives for biological control, emerging clinical importance and recent advances in genetics. Curr. Genet. 59,73-90.

Paraszkiewicz K., Moryl M., Płaza G., Bhagat D., Satpute S., Bernat P. 2019. Surfactants of microbial origin as antibiofilm agents. Int. J. Environ. Health Res. 1, 1-20.

Passoth V., Fredlund E., Druvefors U.Ä., Schnürer J. 2006. Biotechnology, physiology and genetics of the yeast Pichia anomala. FEMS Yeast Res. 6, 3-13.

Piano S.V., Neyrotti Q., Migheli M.L. 1997, Biocontrol capability of Metschnikowia pulcherrima against Botrytis postharvest rot of apple, Postharvest Biol. Technol. 11, 131-140.

Polonelli L., Magliani W., Ciociola T., Giovati L., Conti S. 2011. From Pichia anomala killer toxin through killer antibodies to killer peptides for a comprehensive anti-infective strategy. Antonie Van Leeuwenhoek 99, 35-41.

Poomtien J., Thaniyavarn J., Pinphanichakarn P., Jindamorakot S., Morikawa M. 2013. Production and characterization of a biosurfactant from Cyberlindnera samutprakarnensis jp52t. Biosci. Biotechnol. Biochem. 77, 2362-2370.

Rodríguez-Cousiño N., Maqueda M., Ambrona J., Zamora E., Esteban R., Ramírez M. 2011. A new wine Saccharomyces cerevisiae killer toxin (Klus), encoded by a double-stranded RNA virus, with broad antifungal activity is evolutionarily related to a chromosomal host gene. Appl. Environ. Microbiol. 77, 1822-1832.

Romanazzi G., Smilanick J.L., Feliziani E., Droby S. 2016. Integrated management of postharvest gray mold on fruit crops. Postharvest Biol. Technol. 113, 69-76.

Rufino R.D., Luna J.M., Sarubbo L.A., Rodrigues L.R.M., Teixeira J.A.C., Campos-Takaki G.M. 2011. Antimicrobial and anti-adhesive potential of a biosurfactant Rufisan produced by Candida lipolytica UCP 0988. Colloids Surf. B. 84, 1-5.

Sanada H., Nakagami G., Takehara K., Goto T., Ishii N., Yoshida S., Tsunemi Y. 2014. Antifungal effect of non-woven textiles containing polyhexamethylene biguanide with sophorolipid: a potential method for Tinea pedis prevention. Healthcare 2, 183-191.

Saravanakumar D., A. Ciavorella D. Spadaro A. Garibaldi M.L. 2008. Metschnikowia pulcherrima strain MACH1 outcompetes Botrytis cinerea, Alternaria alternata and Penicillium expansum in apples through iron depletion, Postharvest Biol. Technol. 49, 121-128.

Satputea S., Banpurkar A., Banat I., Sangshetti J., Patil R., Gade W. 2016. Multiple roles of biosurfactants in biofilms. Curr. Pharm. Des. 22, 1429-1448.
Sipiczki M. 2006. Metschnikowia strains isolated from botrytized grapes antagonize fungal and bacterial growth by iron depletion, Appl. Env. Microbiol. 72, 6716-6724.

Spadaro D., Vola S., Piano M.L. 2002. Mechanisms of action and efficacy of four isolates of the yeast Metschnikowia pulcherrima active against postharvest pathogens on apples. Postharvest Biol. Technol. 24, 123-134.

Tang W., Wang Y., Zhang J., Cai Y., He Z. 2019. Biosynthetic pathway of carotenoids in Rhodotorula and strategies for enhanced their production. $J$. Microbiol Biotechnol. 29, 507-517.

Thaniyavarn J., Chianguthai T., Sangvanich P., Roongsawang N., Washio K., Morikawa M., Thaniyavarn S. 2008. Production of sophorolipid biosurfactant by Pichia anomala. Biosci. Biotechnol. Biochem. 72, 2061-2068.

Yang Q., Zhang H., Zhang X., Zheng X., Qian J. 2015. Phytic acid enhances biocontrol activity of Rhodotorula mucilaginosa against Penicillium expansum contamination and patulin production in apples. Front. Microbiol. 6, 1296-1310.

Yao H., Tian S., Wang Y. 2004. Sodium bicarbonate enhances biocontrol efficacy of yeasts on fungal spoilage of pears. Int. J. Food Microbiol. 93, 297-304.

Younis G., Awad A., Dawod R.E., Yousef N.E. 2017. Antimicrobial activity of yeasts against some pathogenic bacteria. Vet World. 10, 979-983.

Zhang D., Spadaro D., Valente S., Garibaldi A., Gullino M.L. 2012. Cloning, characterization, expression and antifungal activity of an alkaline serine protease of Aureobasidium pullulans PL5 involved in the biological control of postharvest pathogens. Int. J. Food Microbiol. 153, 453-464.

Zhang H., Wang L., Dong Y., Jiang S., Cao J., Meng R. 2007. Postharvest biological control of gray mold decay of strawberry with Rhodotorula glutinis. Biol. Control. 40, 287-292.

Zhang H., Wang L., Ma L., Dong Y., Jiang S., Xu B., Zheng X. 2009. Biocontrol of major postharvest pathogens on apple using Rhodotorula glutinis and its effects on postharvest quality parameters. Biol. Control 48, 79-83.

Zhang H., Yang Q., Ge L., Zhang G., Zhang X. 2016. Chitin enhances biocontrol of Rhodotorula mucilaginosa to postharvest decay of peaches. Int. J. Biol. Macromol. 88, 465-475.

Zhao Y., Tu K., Shao X., Jing W., Su Z. 2008. Effects of the yeast Pichia guilliermondii against Rhizopus nigricans on tomato fruit. Postharvest Biol. Technol. 49, 113-120. 